\title{
OCORRÊNCIA DE REAÇÃO SOROLÓGICA CONTRA SALM ONELLA PULLORUM EM AVESDE “FUNDO DE QUINTAL" DO ESTADO DE SÃO PAULO, BRASIL
}

\author{
F.G . Buchala'1, M .M. Ishizuka², L.A. M athias³ , A. Berchieri Júnior³, A.G .M. Castro4, \\ A.L.S.P. Cardoso ${ }^{4}$, E.N .C. Tessari ${ }^{4}$, A .M .I. Kanashiro ${ }^{4}$
}

${ }^{1}$ Coordenadoria de Defesa Agropecuária, Av. Brasil, 2340, CEP 13073-001, Campinas, SP, Brasil.

RESUMO

\begin{abstract}
Esta pesquisa objetivou demonstrar a ocorrência de reações sorológicas contraSalmonellaspp. nas populações de aves de explorações não tecnificadas e com finalidade de subsistência, em propriedadesruraislocalizadasemáreasgeográficaspróximasagranjas dereprodutoras (matrizeiros) do Estado de São Paulo. Foram sel ecionadas três granjas de matrizes, oficialmente reconhecidas como sendo livres deS. Pullorum (SP), S. Gallinarum(SG),S. Enteritidis (SE),S. Typhimurium(ST), e15 criações vizinhas, cuja população deaves foi amostrada com col heita e processamento de 406 sorossangüíneos. O diagnóstico foi real izado pelatécnicadesoroaglutinação rápidaem placaelenta em tubos. A freqüência encontrada foi igual a $73 \%$ de propriedades com aves sororeagentes ao antígeno testado de SP (teste da pul orose). A ocorrência observada de aves sororeagentes foi de $16,5 \%$. Os dados obtidos sugerem queo agenteestudado está amplamentedifundido nas criações informais deaves de "fundo dequintal", colocando em risco constanteos criatórios deexploração industrial, os quais necessitam adotar emanter boas práticas de biosseguridade para preservar a integridadesanitáriadosplantéis.
\end{abstract}

PALAVRAS-CHAVE: Pulorose, biosseguridade, doenças, epidemiologia, infecções, sanidade.

\section{ABSTRACT}

OCCURRENCE OF SEROLOGIC RESPONSES AGAINST SALM ONELLA PULLORUM IN DOMESTIC BACKYARD POULTRY IN THE STATE OF SÃ O PAULO, BRAZIL. This investigation ai med to demonstratetheoccurrenceof serological reactionstoSalmonel laspp. in backyard domestic poultry for privateconsumption located nextto parent flocksin theStateof São Paulo. Threeparent flocks officially recognised as freefromS. Pullorum (SP), S. Gall linarum (SG), S. Enteritidis (SE),S. Typhimurium(ST) and 15neighboring backyard flockswereselected. A sampleof 406chickensfrom thebackyard flockswas bled, and thediagnosticwas carried out by theagglutination tests in plate and in tubes. Thefrequency found was $73 \%$ of flocks with chickens reacting to the antigens of SP. The frequency of reacting chickens was $16.5 \%$ for the antigen of SP. The results show that the etiol ogical agentstudied is widespread among thebackyard flocks, posing a constant risk for the commercial poultry flocksthatneed to adoptand keep good biosecurity practicesto preservetheir health status.

KEY WORDS: Pullorum disease, biosecurity, diseases, epidemiology, infections, animal health.

\section{INTRODUÇÃO}

A conquistae o reconhecimento destatus sanitário de estabelecimento livre de doenças contempladas pelo ProgramaNacional deSanidadeA vícola(BRASIL, 1994) envolvem el evados investimentos em aspectos relativos aos recursos físicos, humanos e de monitoramento laboratorial representado por uma sistemática colheita, anál ise einterpretação deresultados deprovas diagnósticas. Essa condição deveser mantidapor estratégias deprofilaxiaquevisemimpedir a entrada de agentes etiológicos de doenças e, no caso deocorrência, essas medidas devem ser capazes de detectá-los precocemente, iniciando-se imediatamente ações de erradicação. Essas medidas, aplicadas de forma planejada, organizada e devidamente

2Universidadede São Paulo, Faculdadede Medicina Veterinária eZootecnia, São Paulo, SP, Brasil.

${ }^{3}$ UniversidadeEstadual Paulista, FaculdadedeCiências A grarias eV eterinárias, Jaboticabal, SP, Brasil.

${ }^{4}$ Instituto Biológico, Centro A vançado dePesquisa Tecnológica do A gronegócio A vícola, Descalvado, SP, Brasil. 
supervisionadas, permitem manter a situação sanitáriaarduamenteconquistada por cadaestabelecimento avícola, contribuindo para o desenvolvimento da economia nacional.

Asprincipaisinfecções quecomprometemaavicultura comercial são as salmoneloses: Salmonella enterica subspécie enterica sorovar Pullorum (SP),S. enterica subspécieenterica sorovar Gallinarum (SG), Salmonella enterica subspécie enterica sorovar Typhimurium (ST), Salmonella enterica subspécie entericasorovar Enteritidis(SE) easmicoplasmoses (M ycoplasma gallisepticum e M ycoplasma synoviae), razão pela qual são contempladas pelo Programa $\mathrm{N}$ acional de Sanidade A vícola. A respectiva legislação estabel ecequeempresasavícolas, matrizeiras e avozeiras (multiplicadoras genéticas) devem estar obrigatoriamente livres despas êf erpajądes

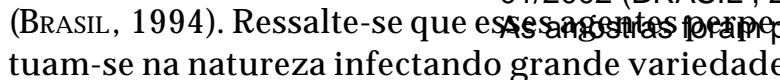
deaves domésticas e devida livre, notadamentena ausência demanifestação clínica, edisseminam-se na população de aves, por mecanismos de transmissão horizontal e vertical (BERCHIERI JúNIOR, 1997). A adoção de medidas profiláticas em granjas é importanteem faceda omissão dalegislação no que diz respeito ao control esanitário em aves comerciais (frango decorteegal inha de postura) eem aves domésticas de vida livre. A ssim, a estratégia governamental e empresarial adotada para reduzir a ocorrência dessas infecções nos produtos comerciaiséa manutenção dosplantéisreprodutoreslivres desses patógenos (N A scım ENToet al ., 1997), responsáveis por elevado impacto econômico e danos à saúde dos consumidores (PANNETA, 1999) quando estiverem envolvidas principalmente a SE ea ST.

Wray \& DAvies (1994) mencionam que:

1. O controle das doenças causadas por SP eSG, principais patógenos de interesse para a indústria avícola, foi al cançado em muitospaíses pelaintrodução de medidas de profilaxia seguida de ações de vigilância epidemiológica representadaspor exames sorológicos e sacrifício compulsório de plantéis infectados.

2. O teste desoroaglutinação que utiliza antígeno corado esanguetotal temsido empregado comsucesso, por mais demeio século, na identificação delotes de aves infectadas por SP ou SG. Face ao compartilhamento do antígeno somático do grupo $D$ entre SE, SG e SP, é possível o emprego do teste de aglutinação para o diagnóstico das salmoneloses mencionadas.

3. A s características do teste que emprega sangue são: (i) éuma prova rápi da eque podeser aplicada no campo, é pouco dispendiosa, requer pouco equipamento e só exige separação do soro se for realizada em laboratório; (ii) a especificidade é baixa quando a provaéreal izada com sanguetotal e requer experiência para não registrar resultados falsos.

O objetivo desta pesquisa é verificar a ocorrência de respostas sorológicas ao antígeno de SalmonellaPullorum(testedepuloroseprovasrápida e lenta) em populações de aves domésticas de "fundo de quintal", presentes em propriedades ruraisvizinhas degranjas demultiplicação genética. Desta forma, pode-sedeterminar o risco deintrodução deagentes dedoenças transmissíveis distribuídos na natureza.

\section{MATERIAL E MÉTODOS}

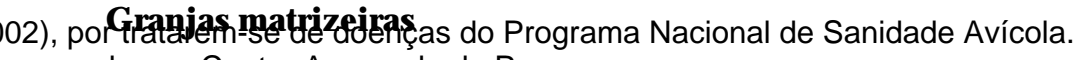
sadas no Centro Avançado de Pes

Foramselecionadas 3granjasdematrizes(Filial 1, Filial 2eFilial 3), local izadas noEstado deSão Paulo, pertencentes a uma mesma empresa, integrantes do Programa Nacional deSanidade A vícola, com status sanitário oficialmentereconhecido como sendolivres desalmonelosesemicoplasmoses, conformedemonstraram as colheitas deamostras seqüenciais durante os anos de 1998, 1999 e 2000.

\section{Criações de subsistência (aves caipi ras)}

Foram examinadas 15 criações vizinhas, sendo 7, 5 e 3, respectivamente, localizadas ao redor dos matrizeiros: Filial 1, Filial 2 e Filial 3.

\section{A mostragem (número de aves caipiras selecionadas)}

Optou-se por utilizar o val or da prevalência estimadadapulorose, ecalculou-seadimensão daamostra para uma precisão da estimativaigual a $10 \%$, erro de primeira espécie $(\alpha)$ igual a $5 \%$ e com a devida correção para população de tamanho conhecido (JeNICEK;Cléroux, 1987). NaTabela lestãoapresentados os tamanhos das amostras obtidas de cada criação e a respectiva população no momento da amostragem em relação a cada matrizeiro.

\section{A mostras de sangue}

A colheita o acondicionamento e a remessa ao laboratório atenderamal nstrução $\mathrm{N}$ ormativan이 de 09/ 01/ 2002 (BRASIL, 2002), por tratarem-se de doenças do Programa N acional de Sanidade A vícola. As amostras foram processadas no Centro $A$ vançado de Pesquisa Tecnológica do Agronegócio Avícola (CAPTAA) do Instituto Biológico do Estado de São Paulo. 
Tabela 1 - Matrizeiro e respectivas criações vizinhas, segund o otamanho da população deaves ea amostragem da amostra de aves de "fundo de quintal". Estado de São Paulo, 2000.

\begin{tabular}{lccc}
\hline Matrizeiro & Criação & $\begin{array}{c}\text { No aves } \\
\text { existentes }\end{array}$ & $\begin{array}{c}\text { No aves na } \\
\text { amostra }\end{array}$ \\
\hline 1A & 38 & 28 \\
Filial 1 & 1 B & 60 & 39 \\
(360.000 aves) & 1 C & 47 & 33 \\
& 1E & 48 & 34 \\
& 1F & 34 & 26 \\
\hline Subtotal & 1G & 41 & 14 \\
\hline & & 284 & 204 \\
\hline Filial 2 & 2A & 15 & 13 \\
(7.000 aves) & 2B & 37 & 29 \\
& 2 C & 24 & 20 \\
\hline Subtotal & 2 D & 62 & 40 \\
\hline Filial 3 & 2 & 30 & 24 \\
(13.000 aves) & 3A & 168 & 126 \\
\hline Subtotal & 3 C & 100 & 24 \\
\hline Total & & 25 & 20 \\
\hline & & 155 & 97 \\
\hline
\end{tabular}

Provas de soroaglutinaçao para detecção de anticorpos contra SP (teste de pulorose)

O procedimento seguiu aqueleindicado pelo Programa Nacional de Sanidade A vícola (BrASIL, 1995). A s amostras foram inativadas em banho-maria a $56^{\circ}$ C. Para os testes desoroaglutinação rápida em placa, utilizou-se antígeno corado comercial Intervet International (Boxmeer-Holanda). Foram distribuídos $30 \mu \mathrm{L}$ de soro e $30 \mu \mathrm{L}$ de antígeno em placas de vidro, homogeneizou-se a mistura por no máximo 2 minutos verificando-se a formação de grumos indicando a reação antígeno-anticorpo. Os soros positivos foram então submetidos a soroaglutinação lenta em tubos, para qual utilizou-se o antígeno diluído 1:20 em salina tamponada fenolizada. Distribuiu-se $1 \mathrm{~mL}$ dessa solução em tuboseacrescentou-se80uLdo soro. Os tubos foram incubados a $37 \circ \mathrm{C} / 48 \mathrm{~h}$. Foram considerados positivos os tubos onde ocorreu precipitaçãodocomplexo antígeno-anticorpo econseqüentemente transparência da solução.

\section{M étodo estatístico}

Estimativa da freqüência de ocorrência (proporção) desalmonelose por intervalo de confiança, com 95\% deprobabilidade. Utilizou-seotestedadiferença entreduasproporçõescomaproximaçãonormal (LESER et al., 1973).

\section{RESULTADOS}

Freqüência de criatórios de aves de "fundo de quintal " sororeagentes ao antígeno testado

Entreos 15criatóriosestudadosquepossuemaves de "fundo de quintal", 11 apresentaram pelo menos umaavesororeagenteparao antígeno deSP, resultando em freqüênciaigual a $73 \%$ dos criatórios comaves sororeagentes; a inferência por intervalo para $95 \%$ de confiança foi igual à LC (50,5\% I_— I 95,5\%).

\section{Freqüência de aves sororeagentes ao antígeno} de SP

Tabela 2- Resultados positivos desorologia paraS. Pullorum, segundo o matrizeiroeas respectivascriações de"fundo de quintal". Estado deSão Paulo, 2000.

\begin{tabular}{|c|c|c|c|c|}
\hline Matrizeiro & $\begin{array}{l}\text { Criação "fundo } \\
\text { dequintal" }\end{array}$ & $\begin{array}{c}\text { No total de } \\
\text { Aves examinadas }\end{array}$ & $\begin{array}{c}\text { No de aves } \\
\text { positivas }\end{array}$ & $\begin{array}{c}\% \text { deaves } \\
\text { positivas }\end{array}$ \\
\hline & $1 \mathrm{~A}$ & 16 & 5 & 31,2 \\
\hline & $1 \mathrm{~B}$ & 39 & 6 & 15,4 \\
\hline & $1 \mathrm{C}$ & 33 & 0 & 0,0 \\
\hline \multirow[t]{4}{*}{ Filial 1} & $1 \mathrm{D}$ & 34 & 10 & 29,4 \\
\hline & $1 \mathrm{E}$ & 26 & 3 & 10,3 \\
\hline & $1 \mathrm{~F}$ & 14 & 4 & 28,6 \\
\hline & $1 G$ & 30 & 2 & 6,7 \\
\hline \multirow[t]{3}{*}{ Subtotal } & & 204 & 30 & 14,7 \\
\hline & $2 \mathrm{~A}$ & 13 & 0 & 0,0 \\
\hline & $2 \mathrm{~B}$ & 29 & 0 & 0,0 \\
\hline
\end{tabular}

Continua 
Tabela 2 - Continuação.

\begin{tabular}{lcccc}
\hline Matrizeiro & $\begin{array}{c}\text { Criação "fundo } \\
\text { dequintal" }\end{array}$ & $\begin{array}{c}\text { No total de } \\
\text { Aves examinadas }\end{array}$ & $\begin{array}{c}\text { No de aves } \\
\text { positivas }\end{array}$ & $\begin{array}{r}\text { \% de aves } \\
\text { positivas }\end{array}$ \\
\hline Filial 2 & 2 C & 20 & 4 & 20,0 \\
& 2D & 20 & 0 & 0,0 \\
& 2E & 24 & 5 & 20,8 \\
\hline Subtotal & & 105 & 9 & 8,6 \\
\hline \multirow{2}{*}{ Filial 3 } & 3A & 24 & 14 & 58,3 \\
& 3B & 53 & 8 & 15,1 \\
\hline Subtotal & 3C & 20 & 6 & 30,0 \\
\hline Total & & 97 & 28 & 28,0 \\
\hline
\end{tabular}

Das 406 aves examinadas, 67 foram positivas à sorologia para SP (teste de pulorose), representando $16,5 \%$ deaves sororreagentes e um intervalo de confiança para a inferência com $95 \%$ de confiança igual à LC (12,9\%|-- | 20,1\%). Os resultados observados, segundo a empresa de multiplicação genética (matrizeiro) eas propriedadescomaves de" fundo de quintal" circunvizinhas, encontram-se reunidos na Tabela 2.

\section{DISCUSSÃO}

A avaliação dapresençadefontes deinfecção pela detecção deanticorpos representa um rápido eprático instrumento de importância epidemiológica, por revelar contacto das aves com os respectivos agentes, demonstrando a presença ou a circulação deagentes etiológicos na população (WRAY \& DAVIES, 1994).

A observação de 73,0\%, LC (50,5\% I-— I 95,5\%) de criatórios com pelo menos duas aves sororeagentes para SP indica elevada freqüência de circulação da bactéria entre essas aves, devido a multiplicidade de fatores de transmissão horizontal, essa bactéria pode romper a barreiradabiosseguridadeeinfectar osl otes demultiplicação genética, como relatado por BERCHIERIJÚNIOR (1997).

\section{CONCLUSÕES}

Os resultados obtidos no presente estudo permitem concluir que:

1. A freqüênciadecriatórioscomavessororeagentes ao antígeno de SP foi igual a $73 \%$.

2. A freqüência deaves sororeagentes ao antígeno deSP foi igual a $16,5 \%$.

3. A elevada freqüência de criatórios com aves sororeagentesao antígenotestado sugerequeoagente SP encontra-seamplamentedistribuído na natureza.
4. A atual legislação, omissa no controlesanitário em aves domésticas de "fundo de quintal", deve ser revisada pelosórgãos competentes de DefesaSanitária Animal e contemplar um programa de saúde animal mais amplo, capaz de garantir eassegurar a exploração avícolaemescalaindustrial, preservando os plantéis de multiplicação genética como sendo livres dos patógenos responsáveis por enfermidades querepresentamelevado impacto econômicoepossíveis danos à saúde dos consumidores.

\section{REFERÊNCIAS}

BERCHIERI JUNIOR, A. Doenças de transmissão vertical. In: SIMPÓSIO TÉCNICO DE PRODUÇÃO DE OVOS, 7., 1997, Campinas. A nais. São Paulo: APA, 1997. p.133142.

Brasil. Ministério da A gricultura, do A bastecimento e da Reforma A grária. Programa N acional de Sanidade A vícola. A toslegais. Portarian ${ }^{\circ} 193$ de 19 desetembro de 1994. D iário O ficial da República Federativa do Brasil, Poder Executivo, Brasília-DF, 22 desetembro de1994. Seção 1, p.14309-14312.

BrASIL. Ministério da A gricultura, do A bastecimento eda Reforma Agrária. Normas de Credenciamento e Monitoramento de Laboratórios deDiagnóstico das SalmonelosesA viárias(S. Enteritidis, S. Gallinarum, S. PullorumeS. Typhimurium). Portarian ${ }^{\circ} 126$, de03de novembro de 1995. D iário O ficial daR epública Federativa do Brasil, Poder Executivo, Brasília-DF, 06denovembro de 1995. Seção 1, p.17694-17698.

BRASIL. M inistério da Agricultura, Pecuária eA bastecimento. Normas Técnicas para Controle e Certificação deN úcl eos eEstabel ecimentos A vícolas como livres de Salmonella Gallinarum e Salmonella Pullorum e livres ou controlados para Salmonella Enteritidis eSal monella Typhimurium. A toslegais. Instrução N ormativa no03de09 dejaneiro de2002. D iário 0 ficial da República Federativa do Brasil, Poder Executivo, Brasília-DF, 16dejaneiro de2002. Seção 1, p.14-18. 
Jenicek, M.\& Cléroux, R.É pidemiologie: principies, techniques, applications. Quebec: Edisem, 1987. 454p.

Leser, W., Ribeiro Netto,A.;Gsaw, O.A.;M arlet, J.M.Elementos deestatística para a área deciências da saúde. SãoPaulo: Escola Paulista de MedicinaUN IFESP, 1973. 180p.

Nascimento, V.P.; M oraes, H.L.S.;RIBeiRo,A.R.;SAntos,L.R.; Cardoso, M.O.; Pontes, A.P.; Oliveira, S.D. Aspectos favoráveisedesfavoráveis dos programasdevacinação no controlesanitário dasal monelose. In:SIMPÓSIO TÉCN ICO DE PRODUÇÃ O DE OVOS, 7., 1997, Campinas. A nais. São Paulo: APA, 1997. p.143-152.

PANNETA, J.C. Segurançaalimentar eHACCP na produção deovos. In:CONGRESSODEPRODUÇÃ OECONSUMO DE OVOS, 1., 1999, São Paulo. A nais. São Paulo: APA, 1999. p.71-78.
Wray, C. \& Davies, R.H. Guidelines on detection and monitoring of salmonella infected poultry flocks with particular reference to Salmonella enteritidis. In: REPORT OF A WHO CONSULTATION ON STRATEGIESFORDETECTION ANDMONITORING OF SALMONELLA INFECTED POULTRY FLOCKS, Austria: WHO - V eterinary P ublic H ealth U nit, 1994. p.29-34.

Recebido em 8/ 12/ 05 A ceito em 29/ 02/ 06 